\title{
Quantitative genotyping of single-nucleotide polymorphisms by allele-specific oligonucleotide hybridization on DNA microarrays
}

\author{
Andreas M. Rickert*, Agim Ballvora*l, Ulrich Matzner†, Manfred Klemm† \\ and Christiane Gebhardt*
}

*Max-Planck-Institute for Breeding Research, Carl-von-Linne-Weg 10, 50829 Cologne, Germany, and †Alpha Technology GmbH, Hürth, Germany

Genotyping of SNPs (single-nucleotide polymorphisms) has challenged the development of several novel techniques [Twyman and Primrose (2003) Pharmacogenomics 4, 67-79]. Most of these methods have been introduced to discriminate binary SNPs in diploid species. In the present study, the quantitative genotyping of SNPs in natural DNA pools of a polyploid organism via DNA microarrays was analysed. Three randomly selected SNP loci were genotyped in the tetraploid species potato (Solanum tuberosum). For each SNP, 24 oligomers were designed, 12 with forward and 12 with reverse orientation. They contained the polymorphic site at one of the positions II, I4 and I7. Several steps of optimizations were performed, including the 'materials' used and the establishment of hybridization conditions. Glass surfaces were either epoxy- or aldehyde-modified, and allele-specific oligonucleotides contained either $\mathrm{SH}$ or $\mathrm{NH}_{2}$ groups. Hybridization stringency conditions were established by varying the concentration of formamide in the hybridization buffer. For SNP BA2I3cl4t7/403, the quantitative discrimination between all four different naturally occurring genotypes could be demonstrated.

\section{Introduction}

SNPs (single-nucleotide polymorphisms) represent the most common type of genetic variations accounting for approx. $90 \%$ of all polymorphisms [2]. As a result of genome sequencing efforts, large number of SNPs have been discovered, not only in humans [3] (see also the SNP Consortium LTP at http//snp.cshl.org), but also in other diploid organisms such as Drosophila melanogaster (fruitfly or vinegar fly) [5], Arabidopsis thaliana (thale cress) [6] and cereal species [7], and recently in the polyploid potato Solanum tuberosum [8]. Owing to their direct accessibility, SNPs have become popular markers for a wide range of genetic applications, and a number of different SNP genotyping methods have been introduced. However, no single method has been widely accepted [1,9-14]. For linkage and association studies, many SNP markers have to be scored in large number of individuals. Since this procedure is timeconsuming and costly, an alternative approach is to pool samples of affected and unaffected individuals and subsequently estimate, quantitatively, allele frequencies in these subsets. To attain this goal, several SNP genotyping methods have been evaluated [15-28]. Although ASO (allele-specific oligonucleotide) hybridization on microarrays is among the most promising techniques for genotyping individual SNPs [6,29], documented studies of its applicability for quantification of allele frequencies in DNA pools are lacking. In the present paper we describe quantitative genotyping using microarray-based ASO hybridization of three binary SNPs in different homozygous and heterozygous tetraploid potatoes. These genotypes represent natural pools of four alleles for which five allelic ratios are distinguishable: 4:0, 3:I, $2: 2,1: 3$ and $0: 4$ [25].

\section{Materials and methods}

\section{Plant material}

FI plants of a cross between two tetraploid potato cultivars SRII and SRI2 were provided by SAKA-RAGIS (Pflanzenzucht GbR, Windeby, Germany) [8]. FI plants that had been evaluated by pyrosequencing or SNuPE (single nucleotide primer extension) combined with DHPLC (denaturing high-performance liquid chromatography) for the test of SNPs and represented the different allele dosages, were selected for the evaluation of quantitative accuracy of allele-specific hybridization on microarrays. Genomic DNA was isolated from leaf tissue as described previously [30].

\section{SNP markers}

The SNPs BA44al0t7//74, BA2/3c|4t3/268+269 and $\mathrm{BA} 2$ | $3 \mathrm{c} \mid 4 \mathrm{t} 7 / 403$ have been identified by comparative Sanger

\footnotetext{
Key words: allele-specific-oligonucleotide, DNA-microarray, locus, polyploidy, quantitative genotyping, single-nucleotide polymorphism.

Abbreviations used: ASO, allele-specific oligonucleotide; DHPLC, denaturing high-performance liquid chromatography; SNP, single-nucleotide polymorphism; SNuPE, single nucleotide primer extension. To whom correspondence should be addressed (email ballvora@mpiz-koeln.mpg.de).
} 


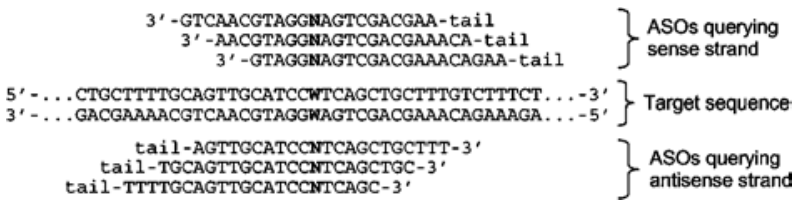

Figure I Schematic representation of the panel of ASOs designed for

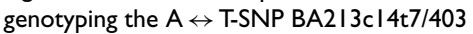

The set of ASOs for allele-specific hybridization comprises 24 different 22-meric oligonucleotides querying the sense as well as the antisense strand of the PCR-amplified target region including the SNP. ASO probes are walking over the polymorphic sites presenting the allelic nucleotide at position II, I4 or 17 from the $5^{\prime}$-end. For each of these ASOs, four different versions were designed, carrying all four bases at the SNP position. ASOs are attached to tails composed of a $\mathrm{C}_{6}$ linker and an $\mathrm{SH}$ or $\mathrm{NH}_{2}$ group. $\mathrm{N}, \mathrm{A} / \mathrm{T} / \mathrm{G} / \mathrm{C} ; \mathrm{W}, \mathrm{A} / \mathrm{T}$.

DNA sequencing and can be accessed through the website http://gabi.rzpd.de/PoMaMo.html [8]. The precise allele dosages of SNPs in the different genotypes were confirmed by pyrosequencing [25] or by SNuPE in combination with DHPLC as described previously [3I].

\section{Amplification and labelling of target sequences}

For amplification and labelling of target sequences (lengths between 180 and $216 \mathrm{bp}$ ), the following primer combinations were used: BA44a l 0t7Fal (5'-CTACATCACCTGCAGCACTCC-3') and BA44al0t7Ral (5'-TTTCATGGAAGCTGGACGGCTAG-3'); BA2 $13 \mathrm{c}|4 \mathrm{t} 3 \mathrm{Ca}|$ (5'-CGGTCAGGGACATCATGCGCA-3') and BA2I3cl4t3Dal (5'-GTGGTATCTGCTAGCAATTGCTAC-3'); BA2 I 3c $14 \mathrm{t} 7 \mathrm{Ba} \mid \mathrm{rev}$ (5'-TCTAACCTGGGCAGTCAGAGAC-3') and BA2।3cl 4t7B (5'-TCTTGACGCAAACCTCTGCGAG-3'). Primers were purchased from Metabion (Martinsried, Germany). PCRs were performed as described in [25]. Aliquots (30 ng) of the initial PCR amplifications were subjected to a second round of PCR containing $20 \mu \mathrm{M}$ Cy3 (indocarbocyamine)dUTP (Amersham Biosciences, Freiburg, Germany) and $80 \mu \mathrm{M}$ dTTP. PCR products were purified using a PCR purification kit (Qiagen, Hilden, Germany) according to the manufacturer's instructions.

\section{Preparation of arrays}

For each SNP, 24 different 22-meric oligonucleotides were designed: 12 for the forward-strand sequence and 12 for the reverse-strand sequence. All four bases were represented at the polymorphic site, which was at position II, 14 or 17 from the 5 -end (Figure I). Each oligonucleotide probe (10$20 \mu \mathrm{M}$ solution) was spotted in triplicate/slide using the spotting device BioGrid (BioRobotics, Oxford, U.K.). The probes were covalently attached to the glass slides (Quantifoil, Jena, Germany) following the manufacturer's instructions. The surfaces of the slides were either epoxymodified or aldehyde-modified. The probe-loaded slides were blocked directly before hybridization following the manufacturer's (Quantifoil) instructions.

The ASOs had $\mathrm{C}_{6}$ linkers at their $5^{\prime}$-ends and were either $\mathrm{SH}$ - or $\mathrm{NH}_{2}$-modified, and were purchased from Interactiva Biotechnologie $\mathrm{GmbH}$ (Ulm, Germany).

\section{Hybridization and washing of arrays}

Labelled PCR product $(20 \mu \mathrm{l})$ from the targeted locus was mixed with $180 \mu \mathrm{l}$ of hybridization buffer $[10 \mathrm{mM}$ Tris $/ \mathrm{HCl}$, $\mathrm{pH} 7.5$, I mM EDTA, $0.6 \mathrm{M} \mathrm{NaCl}$, I $\times$ Denhardt's solution ( $0.02 \%$ Ficoll $400,0.02 \%$ polyvinylpyrrolidone and $0.02 \%$ BSA), $0.1 \mathrm{mg} / \mathrm{ml}$ herring sperm DNA and 0-50\% formamide]. Different formamide concentrations $(0,10,20,30$, 40 or $50 \%$ ) were tested to determine the most appropriate stringency conditions. The hybridization cocktail was denatured at $95^{\circ} \mathrm{C}$ for $5 \mathrm{~min}$, cooled on ice, applied to the array using a hybridization frame (GeneScan, Freiburg, Germany) and incubated at $42^{\circ} \mathrm{C}$ for $4 \mathrm{~h}$. Subsequently, the slide was washed for $10 \mathrm{~min}$ with $10 \mathrm{mM}$ Tris $(\mathrm{pH} 7.5)$ and $39 \mathrm{mM}$ $\mathrm{NaCl}$ at $32^{\circ} \mathrm{C}$, and then for 10 min with $10 \mathrm{mM}$ Tris $(\mathrm{pH} 7.5)$ and $19.5 \mathrm{mM} \mathrm{NaCl}$ at $32^{\circ} \mathrm{C}$.

\section{Microarray reading and data analysis}

Arrays were scanned on the GenePixTM 4000B scanner (Axon Instruments, Foster City, CA, U.S.A.) and images were analysed using the software GenePix TM Pro (Axon Instruments).

\section{Results and discussion}

The ASO hybridization on glass slides required several optimization steps [14]. Epoxy- or aldehyde-modified glass surfaces were tested with the same ASOs and hybridization conditions. The signal intensities and signal-to-noise ratios were comparable for both types of slides (results not shown). However, the use of alternative 5'-modifications of ASOs affected the hybridization results substantially. 5'$\mathrm{SH}$-modified oligomers produced up to 35.4-fold stronger signals when compared with the same oligonucleotides having an $\mathrm{NH}_{2}$-modification. No difference in the signal-tonoise ratio was detectable (results not shown).

Owing to the difficulty in predicting hybridization conditions and sequences of ASOs that will allow optimal allele discrimination, these parameters had to be found empirically [10]. To select the appropriate oligonucleotide sequence, the strategy of probe redundancy [29] was chosen. Panels of ASO probes containing the polymorphic site at various positions and querying the forward as well as the reversestrand sequences were used for each SNP (Figure I). In contrast with ASO probes containing the polymorphic site at nucleotide position 17 from the 5 '-end, ASOs with the allelespecific nucleotide at position $1 \mathrm{I}$ or 14 from the 5 '-end 


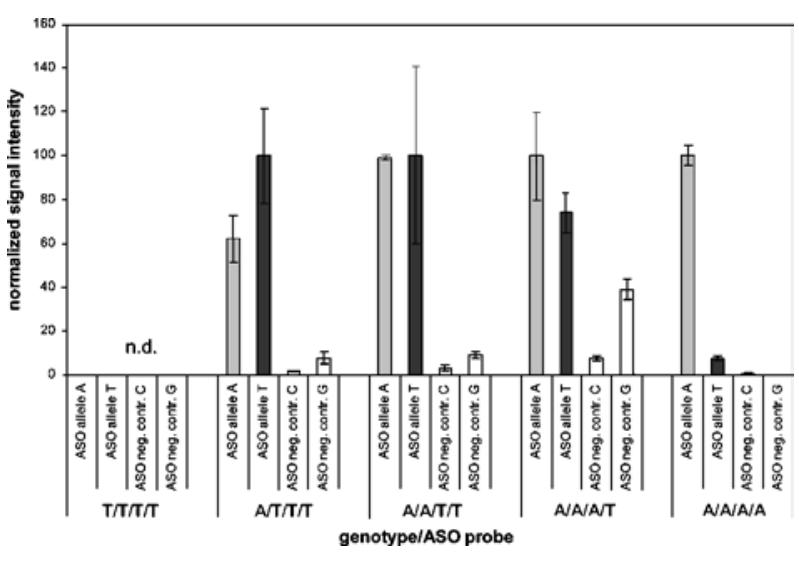

Figure 2 Quantitative genotyping results of four different genotypes of the $\mathrm{A} \leftrightarrow \mathrm{T}$-SNP BA2 I $3 \mathrm{c} \mid 4 \mathrm{t} 7 / 403$

Results are shown for the optimized hybridization condition and ASO probes that allowed the best discrimination between the alleles (hybridization buffer containing $50 \%$ formamide; antisense ASO with polymorphic site at nucleotide position 15 from the 5 '-end). The heights of the histograms correspond to the normalized fluorescence intensities of the spots. S.D. values were calculated on the basis of signal intensities of the three replicas spotted for each ASO probe. The allelic nucleotides are highlighted in black and grey respectively. The genotype $T / T / T / T$ was not available.

were both appropriate to discriminate between different alleles. Sense and antisense oligonucleotides were tested to determine the probe orientation that allowed better allele discrimination. For the SNP locus BA2 |3c|4t7/403, signals of the same quality were obtained with both sense and antisense strands, whereas, in the case of BA2/3c|4t3/ $268+269$, allele discrimination was only possible with the antisense ASOs (results not shown).

The hybridization conditions appeared to be the most important parameter requiring optimization. Several formamide concentrations in the hybridization buffer were tested to achieve a strong and specific hybridization signal with a low background. It turned out that each SNP locus required specific stringency conditions for optimal results. The best hybridization results were obtained using $10 \%(\mathrm{v} / \mathrm{v})$ formamide for BA44a I 0t7/I 74, 30\% formamide for BA2 I 3c I 4t3/ $268+269$ and $50 \%$ formamide for BA2I3cl4t7/403. The requirement to establish specific hybridization conditions for each SNP has been considered as a major drawback of ASO hybridization on microarrays [14].

For SNP loci BA2 I $3 \mathrm{c} \mid 4 \mathrm{t} 3 / 268+269$ and BA2 | $3 \mathrm{c} \mid 4 \mathrm{t} 7 /$ 403 the signal-to-noise ratio was sufficiently low for genotyping the targeted polymorphisms. For SNP BA2।3cl4t7/ 403, all four different genotypes, which were available, could be quantitatively discriminated (Figure 2). In case of BA2 $13 \mathrm{cl} 4 \mathrm{t} 3 / 268+269$, the expected hybridization signals for the two homozygous genotypes were obtained, but the quantitative accuracy failed to distinguish the different allelic ratios of the three heterozygous genotypes (results not shown).
For SNP BA44a 1 0t7/I74, the conditions used were not stringent enough to achieve a reliable genotyping (results not shown). The signals obtained from the negative controls (ASO probes representing non-existing allelic bases) did not allow robust genotyping. For this marker it seems necessary to improve other hybridization conditions such as temperature and, possibly, primer design.

In conclusion, our results suggest that it is possible to perform quantitative genotyping of SNPs using allele-specific hybridization on microarrays. Individual setting of specific hybridization conditions for each SNP and selection of appropriate material are two prerequisites to obtain reliable genotyping results. To our knowledge, this is the first attempt to show the feasibility of the method for quantitative genotyping of SNPs. A comparison of advantages of this method with other techniques will only be possible when more examples will be available. Most of the methods tested so far for quantitative genotyping of SNPs [25] inherit their drawbacks. Therefore the procedure described here remains a promising alternative particularly for simultaneous analysis of a large number of SNPs.

\section{Acknowledgments}

This work was funded by BMBF (Bundesministerium für Bilding und Forschung) grant 03I2290A [GABI (Genome Analysis of the Plant Biological System)]. We thank SAKARAGIS Pflanzenzucht GbR for providing the plants.

\section{References}

I Twyman, R. M. and Primrose, S. B. (2003) Pharmacogenomics 4, 67-79

2 Collins, F. S., Guyer, M. S. and Chakravarti, A. (1997) Science $278,|580-158|$

3 The International SNP Map Working Group (200I) Nature (London) 409, 928-933

4 Reference deleted

5 Long, A. D., Lyman, R. F., Langley, C. H. and Mackay, T. F. C. (1998) Genetics 149, 999-1017

6 Fan, J.-B., Chen, X., Halushka, M. K., Berno, A., Huang, X., Ryder, T., Lipshutz, R. J., Lockhart, D. J. and Chakravarti, A. (2000) Genet. Res. 10, 853-860

7 Buckler, E. S., Thornsberry, J. M. and Kresovich, S. (200I) Genet. Res. 77, 213-218

8 Rickert, A. M., Jeong, H. K., Meyer, S., Nagel, A., Ballvora, A., Oefner, P. J. and Gebhardt, C. (2004) Plant Biotechnol. J. I, 399-410

9 Ellis, M. C. (2000) Genet. Res. 10, 895-897

I0 Gut, I. G. (200 I) Hum. Mutat. 17, 475-492

I I Kwok, P. Y. (2000) Pharmacogenomics I, 23I-235 
12 Kwok, P. Y. (200I) Annu. Rev. Genomics Hum. Genet. 2, 235-258

13 Shi, M. M. (200I) Clin. Chem. 47, 164-172

14 Syänen, A. C. (200I) Nat. Rev. Genet. 2, 940-942

I5 Breen, G., Harold, D., Ralston, S., Shaw, D. and St Clair, D. (2000) Biotechniques 28, 464-470

16 Cho, R. J., Mindrinos, M., Richards, D. R., Sapolsky, R. J., Anderson, M., Drenkard, E., Dewdnay, J., Reuber, L., Stammers, M., Federspiel, N. et al. (1999) Nat. Genet. 23, 203-207

17 Germer, S., Holland, M. J. and Higuchi, R. (2000) Genet. Res. 10, 258-266

18 Giordano, M., Mellai, M., Hoogendoorn, B. and MomiglianoRichiardi, P. (200I) J. Biochem. Biophys. Methods II, $10|-| 10$

19 Gruber, J. D., Colligan, P. B. and Walford, J. K. (2002) Hum. Genet. I I0, 395-40 I

20 Le Hellard, S., Ballereau, S. J., Visscher, P. M., Torrance, H. S., Pinson, J., Morris, S. W., Thomson, M. L., Semple, C. A. M., Muir, W. J., Blackwood, D. H. R. et al. (2002) Nucleic Acid Res. 30, e74

21 Lindroos, K., Sigurdsson, S., Johansson, K., Rönnblom, L. and Syänen, A.-S. (2002) Nucleic Acids Res. 30, e70

22 Matyas, G., Giunta, C., Steinmann, B., Hossle, J. P. and Hellwig, R. (2002) Hum. Mutat. 19, 58-68
23 Norton, N., Williams, N. M., Williams, H. J., Spurlöock, G., Kirov, G., Morrris, D. W., Hoogendoorn, B., Owen, M. J. and O'Donovan, M. C. O. (2002) Hum. Genet. I I0, 47I-478

24 Olsson, C., Liljedahl, U. and Syvänen, A. C. (2003) Methods Mol. Biol. 212, 167-176

25 Rickert, A. M., Premstaller, A., Gebhardt, C. and Oefner, P. J. (2002) Biotechniques 32, 592-593

26 Ross, P., Hall, L. and Haff, S. A. (2000) Biotechniques 29, 620-629

27 Shifman, S., Pisante-Shalom, A., Yakir, B. and Darvasi, A. (2002) Mol. Cell. Probes 16, 429-434

28 Zhang, S., Van Pelt, C. K., Huang, X. and Schulz, G. A. (2002) J. Mass Spectrum 37, 1039-1050

29 Wang, D. G., Fan, J. B., Siao, C. J., Berno, A., Young, P., Sapolsky, R., Ghandour, G., Perkins, N., Winchester, E., Spencer, J. et al. (1998) Science 280, 1077-1082

30 Oberhagemann, P., Chatot-Balandras, C., Bonnel, E., Schäfer-Pregl, R., Wegener, D., Palomino, C., Salamini, F. and Gebhardt, C. (1999) Mol. Breeding 5, 399-4I5

31 Premstaller, A., Oberacher, H., Rickert, A., Huber, C. G. and Oefner, P. J. (2002) Genomics 79, 793-798

Received 2 November 2004/10 February 2005; accepted 25 April 2005 Published as Immediate Publication 25 April 2005, doi:I0.1042/BA20040I77 